\title{
Correction to: EStradiol and PRogesterone in In vitro ferTilization (ESPRIT): a multicenter study evaluating third- versus second-generation estradiol and progesterone immunoassays
}

\author{
N. P. Polyzos ${ }^{1,2,3} \cdot$ E. Anckaert ${ }^{4} \cdot$ P. Drakopoulos ${ }^{5} \cdot$ H. Tournaye ${ }^{5} \cdot$ J. Schiettecatte $^{4} \cdot$ H. Donner ${ }^{6} \cdot$ G. Bobba $^{7} \cdot$ G. Miles $^{8}$. \\ W. D. J. Verhagen-Kamerbeek ${ }^{7}$ E. Bosch ${ }^{9}$
}

Published online: 3 June 2020

(C) Italian Society of Endocrinology (SIE) 2020

\section{Correction to: Journal of Endocrinological Investigation (2020) https://doi.org/10.1007/s40618-020-01211-x}

The article "EStradiol and PRogesterone in In vitro ferTilization (ESPRIT): a multicenter study evaluating third- versus second-generation estradiol and progesterone immunoassays", written by N. P. Polyzos, E. Anckaert, P. Drakopoulos, H. Tournaye, J. Schiettecatte, H. Donner, G. Bobba, G. Miles, W. D. J. Verhagen-Kamerbeek, and E. Bosch was originally published electronically on the publisher's internet portal on 13th March 2020 without open access. With the author(s)' decision to opt for Open Choice the copyright of the article changed on 18th May 2020 to (C) The Author(s) 2020 and the article is forthwith distributed under a Creative Commons Attribution 4.0 International License (https:// creativecommons.org/licenses/by/4.0/), which permits use, sharing, adaptation, distribution and reproduction in any medium or format, as long as you give appropriate credit to

The original article can be found online at https://doi.org/10.1007/ s40618-020-01211-x.

$\triangle$ N. P. Polyzos

nikpol@dexeus.com; n.polyzos@gmail.com

1 Department of Reproductive Medicine, Dexeus University Hospital, Gran Via Carles, 11171-75, 08028 Barcelona, Spain

2 Faculty of Medicine and Pharmacy, Vrije Universiteit Brussel, Laarbeeklaan 101, 1090 Brussels, Belgium

3 Department of Clinical Medicine, Faculty of Health, Aarhus University Incuba/Skejby, Building 2, Palle Juul-Jensens Boulevard 82, Aarhus N, 8200 Aarhus, Denmark

4 Laboratory of Hormonology and Tumour Markers, Universitair Ziekenhuis Brussel, Free University of Brussels, Brussels, Belgium the original author(s) and the source, provide a link to the Creative Commons licence, and indicate if changes were made.

The Original article has been updated.

Publisher's Note Springer Nature remains neutral with regard to jurisdictional claims in published maps and institutional affiliations.
5 Centre for Reproductive Medicine, University Hospital (UZB), Laarbeeklaan 101, 1090 Jette, Brussels, Belgium

6 Medical and Scientific Affairs, Roche Diagnostics GmbH, Nonnenwald 2, 82377 Penzberg, Germany

7 Medical and Scientific Affairs, Roche Diagnostics International Ltd, Forrenstrasse 2, 6343 Rotkreuz, Switzerland

8 Biostatistics and Data Management, Roche Diagnostics Operations Inc., 9115 Hague Road, Building R, Indianapolis, IN 46256, USA

9 Human Reproduction Unit, IVI-RMA, Plaza de la Policia Local 3, 46015 Valencia, Spain 